\title{
Fully Unsupervised Graph-Based Discovery of General-Specific Noun Relationships from Web Corpora Frequency Counts
}

\author{
Gaël Dias \\ HULTIG \\ University of \\ Beira Interior \\ ddg@di.ubi.pt
}

\author{
Raycho Mukelov \\ HULTIG \\ University of \\ Beira Interior \\ raicho@hultig.di.ubi.pt
}

\author{
Guillaume Cleuziou \\ LIFO \\ University of \\ Orléans
}

\begin{abstract}
.
In this paper, we propose a new methodology based on directed graphs and the TextRank algorithm to automatically induce general-specific noun relations from web corpora frequency counts. Different asymmetric association measures are implemented to build the graphs upon which the TextRank algorithm is applied and produces an ordered list of nouns from the most general to the most specific. Experiments are conducted based on the WordNet noun hierarchy and assess $65.69 \%$ of correct word ordering.
\end{abstract}

\section{Introduction}

Taxonomies are crucial for any knowledgebased system. They are in fact important because they allow to structure information, thus fostering their search and reuse. However, it is well known that any knowledge-based system suffers from the so-called knowledge acquisition bottleneck, i.e. the difficulty to actually model the domain in question. As stated in (Caraballo, 1999), WordNet has been an important lexical knowledge base, but it is insufficient for domain specific texts. So, many attempts have been made to automatically produce taxonomies (Grefenstette, 1994), but (Caraballo, 1999) is certainly the first work which proposes a complete overview of the problem by (1) automatically building a hierarchical structure of nouns based on bottom-up clustering methods and (2) labeling the internal nodes of the resulting tree with hypernyms from the nouns clustered underneath by using patterns such as " $\mathrm{B}$ is a kind of A".

(C) 2008. Licensed under the Creative Commons Attribution-Noncommercial-Share Alike 3.0 Unported license (http://creativecommons.org/licenses/by-ncsa/3.0/). Some rights reserved.
In this paper, we are interested in dealing with the second problem of the construction of an organized lexical resource i.e. discovering generalspecific noun relationships, so that correct nouns are chosen to label internal nodes of any hierarchical knowledge base, such as the one proposed in (Dias et al., 2006). Most of the works proposed so far have (1) used predefined patterns or (2) automatically learned these patterns to identify hypernym/hyponym relationships. From the first paradigm, (Hearst, 1992) first identifies a set of lexico-syntactic patterns that are easily recognizable i.e. occur frequently and across text genre boundaries. These can be called seed patterns. Based on these seeds, she proposes a bootstrapping algorithm to semi-automatically acquire new more specific patterns. Similarly, (Caraballo, 1999) uses predefined patterns such as " $\mathrm{X}$ is a kind of Y" or "X, Y, and other Zs" to identify hypernym/hyponym relationships. This approach to information extraction is based on a technique called selective concept extraction as defined by (Riloff, 1993). Selective concept extraction is a form of text skimming that selectively processes relevant text while effectively ignoring surrounding text that is thought to be irrelevant to the domain.

A more challenging task is to automatically learn the relevant patterns for the hypernym/hyponym relationships. In the context of pattern extraction, there exist many approaches as summarized in (Stevenson and Greenwood, 2006). The most well-known work in this area is certainly the one proposed by (Snow et al., 2005) who use machine learning techniques to automatically replace hand-built knowledge. By using dependency path features extracted from parse trees, they introduce a general-purpose formalization and generalization of these patterns. Given a training set of text containing known hypernym pairs, their algorithm automatically extracts useful dependency paths and applies them to new corpora to identify novel pairs. (Sang and Hof- 
mann, 2007) use a similar way as (Snow et al., 2006) to derive extraction patterns for hypernym/hyponym relationships by using web search engine counts from pairs of words encountered in WordNet. However, the most interesting work is certainly proposed by (Bollegala et al., 2007) who extract patterns in two steps. First, they find lexical relationships between synonym pairs based on snippets counts and apply wildcards to generalize the acquired knowledge. Then, they apply a SVM classifier to determine whether a new pair shows a relation of synonymy or not, based on a feature vector of lexical relationships. This technique could be applied to hypernym/hyponym relationships although the authors do not mention it.

On the one hand, links between words that result from manual or semi-automatic acquisition of relevant predicative or discursive patterns (Hearst, 1992; Carballo, 1999) are fine and accurate, but the acquisition of these patterns is a tedious task that requires substantial manual work. On the other hand, works done by (Snow et al., 2005; Snow et al., 2006; Sang and Hofmann, 2007; Bollegala et al., 2007) have proposed methodologies to automatically acquire these patterns mostly based on supervised learning to leverage manual work. However, training sets still need to be built.

Unlike other approaches, we propose an unsupervised methodology which aims at discovering general-specific noun relationships which can be assimilated to hypernym/hyponym relationships detection ${ }^{2}$. The advantages of this approach are clear as it can be applied to any language or any domain without any previous knowledge, based on a simple assumption: specific words tend to attract general words with more strength than the opposite. As (Michelbacher et al., 2007) state: "there is a tendency for a strong forward association from a specific term like adenocarcinoma to the more general term cancer, whereas the association from cancer to adenocarcinoma is weak". Based on this assumption, we propose a methodology based on directed graphs and the TextRank algorithm (Mihalcea and Tarau, 2004) to automatically induce general-specific noun relationships from web corpora frequency counts. Indeed, asymmetry in Natural Language Processing can be seen as a possible reason for

\footnotetext{
${ }^{2}$ We must admit that other kinds of relationships may be covered. For that reason, we will speak about generalspecific relationships instead of hypernym/hyponym relationships.
}

the degree of generality of terms (Michelbacher et al., 2007). So, different asymmetric association measures are implemented to build the graphs upon which the TextRank algorithm is applied and produces an ordered list of nouns, from the most general to the most specific. Experiments have been conducted based on the WordNet noun hierarchy and assessed that $65 \%$ of the words are ordered correctly.

\section{Asymmetric Association Measures}

In (Michelbacher et al., 2007), the authors clearly point at the importance of asymmetry in Natural Language Processing. In particular, we deeply believe that asymmetry is a key factor for discovering the degree of generality of terms. It is cognitively sensible to state that when someone hears about mango, he may induce the properties of a fruit. But, when hearing fruit, more common fruits will be likely to come into mind such as apple or banana. In this case, there exists an oriented association between fruit and mango (mango $\rightarrow$ fruit) which indicates that mango attracts more fruit than fruit attracts mango. As a consequence, fruit is more likely to be a more general term than mango.

Based on this assumption, asymmetric association measures are necessary to induce these associations. (Pecina and Schlesinger, 2006) and (Tan et al., 2004) propose exhaustive lists of association measures from which we present the asymmetric ones that will be used to measure the degree of attractiveness between two nouns, $x$ and $y$, where $f(.,),. P(),. P(.,$.$) and N$ are respectively the frequency function, the marginal probability function, the joint probability function and the total of digrams.

$$
\begin{gathered}
\text { Braun - Blanquet }=\frac{f(x, y)}{\max (f(x, y)+f(x, \bar{y}), f(x, y)+f(\bar{x}, y))} \\
\mathrm{J} \text { measure }=\max \left[\begin{array}{l}
P(x, y) \log \frac{P(y \mid x)}{P(y)}+P(x, \bar{y}) \log \frac{P(\bar{y} \mid x)}{P(\bar{y})}, \\
\left.P(x, y) \log \frac{P(x \mid y)}{P(x)}+P(\bar{x}, y) \log \frac{P(\bar{x} \mid y)}{P(\bar{x})}\right]
\end{array}\right. \\
\text { Confidence }=\max [P(x \mid y), P(y \mid x)] \\
\text { Conviction }=\max \left[\frac{P(x) \cdot P(\bar{y})}{P(x, \bar{y})}, \frac{P(\bar{x}) \cdot P(y)}{P(\bar{x}, y)}\right]
\end{gathered}
$$




$$
\begin{gathered}
\text { Certainty Factor }=\max \left[\frac{P(y \mid x)-P(y)}{1-P(y)}, \frac{P(x \mid y)-P(x)}{1-P(x)}\right] \\
\text { Added Value }=\max [P(y \mid x)-P(y), P(x \mid y)-P(x)]
\end{gathered}
$$

All seven definitions show their asymmetry by evaluating the maximum value between two hypotheses i.e. by evaluating the attraction of $x$ upon $y$ but also the attraction of $y$ upon $x$. As a consequence, the maximum value will decide the direction of the general-specific association i.e. $(x \rightarrow y)$ or $(y \rightarrow x)$.

\section{TextRank Algorithm}

Graph-based ranking algorithms are essentially a way of deciding the importance of a vertex within a graph, based on global information recursively drawn from the entire graph. The basic idea implemented by a graph-based ranking model is that of voting or recommendation. When one vertex links to another one, it is basically casting a vote for that other vertex. The higher the number of votes that are cast for a vertex, the higher the importance of the vertex. Moreover, the importance of the vertex casting the vote determines how important the vote itself is, and this information is also taken into account by the ranking model. Hence, the score associated with a vertex is determined based on the votes that are cast for it, and the score of the vertices casting these votes.

Our intuition of using graph-based ranking algorithms is that more general words will be more likely to have incoming associations as they will be associated to many specific words. On the opposite, specific words will have few incoming associations as they will not attract general words (see Figure 1). As a consequence, the voting paradigm of graph-based ranking algorithms should give more strength to general words than specific ones, i.e. a higher voting score.

For that purpose, we first need to build a directed graph. Informally, if $x$ attracts more $y$ than $y$ attracts $x$, we will draw an edge between $x$ and $y$ as follows $(\mathrm{x} \rightarrow \mathrm{y})$ as we want to give more credits to general words. Formally, we can define a directed graph $\mathrm{G}=(V, E)$ with the set of vertices $V$ (in our case, a set of words) and a set of edges $E$ where $E$ is a subset of $V \times V$ (in our case, defined by the asymmetric association measure value between two words). In Figure 1, we show the directed graph obtained by using the set of words $V=\{$ isometry, rate of growth, growth rate, rate $\}$ randomly extracted from WordNet where rate of growth and growth rate are synonyms, isometry an hyponym of the previous set and rate an hypernym of the same set. The weights associated to the edges have been evaluated by the confidence association measure (Equation 3) based on web search engine counts ${ }^{3}$.

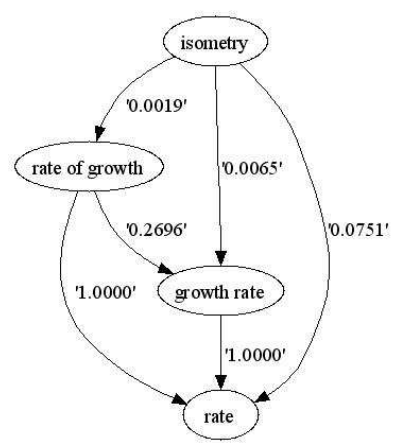

Fig. 1. Directed Graph based on synset \#13153496 (rate of growth, growth rate) and its direct hypernym (rate) and hyponym (isometry).

Figure 1 clearly shows our assumption of generality of terms as the hypernym rate only has incoming edges whereas the hyponym isometry only has outgoing edges. As a consequence, by applying a graph-based ranking algorithm, we aim at producing an ordered list of words from the most general (with the highest value) to the most specific (with the lowest value). For that purpose, we present the TextRank algorithm proposed by (Mihalcea and Tarau, 2004) both for unweighted and weighted directed graphs.

\subsection{Unweighted Directed Graph}

For a given vertex $V_{i}$ let $\operatorname{In}\left(V_{i}\right)$ be the set of vertices that point to it, and let $\operatorname{Out}\left(V_{i}\right)$ be the set of vertices that vertex $V_{i}$ points to. The score of a vertex $V_{i}$ is defined in Equation 8 where $d$ is a damping factor that can be set between 0 and 1 , which has the role of integrating into the model the probability of jumping from a given vertex to another random vertex in the graph ${ }^{4}$.

$$
S\left(V_{i}\right)=(1-d)+d \times \sum_{V j \in \operatorname{In}\left(V_{i}\right)} \frac{1}{\left|\operatorname{Out}\left(V_{j}\right)\right|} \times S\left(V_{j}\right)
$$

\subsection{Weighted Directed Graph}

In order to take into account the edge weights, a new formula is introduced in Equation 9.

\footnotetext{
${ }^{3}$ We used counts returned by http://www.yahoo.com.

${ }^{4} d$ is usually set to 0.85 .
} 


$$
W S\left(V_{i}\right)=(1-d)+d \times \sum_{V j \in \operatorname{In}\left(V_{i}\right)} \frac{w_{j i}}{\sum_{V \in \in u t\left(V_{j}\right)} w_{j k}} \times W S\left(V_{j}\right)
$$

After running the algorithm in both cases, a score is associated to each vertex, which represents the "importance" of the vertex within the graph. Notice that the final values obtained after TextRank runs to completion are not affected by the choice of the initial values randomly assigned to the vertices. Only the number of iterations needed for convergence may be different. As a consequence, after running the TextRank algorithm, in both its configurations, the output is an ordered list of words from the most general one to the most specific one. In table 1, we show both the lists with the weighted and unweighted versions of the TextRank based on the directed graph shown in Figure 1.

\begin{tabular}{|c|c|c|c|c|c|}
\hline \multicolumn{2}{|c|}{ Unweighted } & \multicolumn{2}{c|}{ Weighted } & \multicolumn{2}{c|}{ WordNet } \\
\hline$S\left(V_{i}\right)$ & Word & $W S\left(V_{i}\right)$ & Word & Categ. & Word \\
\hline 0.50 & rate & 0.81 & rate & Hyper. & rate \\
\hline 0.27 & $\begin{array}{c}\text { growth } \\
\text { rate }\end{array}$ & 0.44 & $\begin{array}{c}\text { growth } \\
\text { rate }\end{array}$ & Synset & $\begin{array}{c}\text { growth } \\
\text { rate }\end{array}$ \\
\hline 0.19 & $\begin{array}{c}\text { rate of } \\
\text { growth }\end{array}$ & 0.26 & $\begin{array}{c}\text { rate of } \\
\text { growth }\end{array}$ & Synset & $\begin{array}{c}\text { rate of } \\
\text { growth }\end{array}$ \\
\hline 0.15 & isometry & 0.15 & isometry & Hypo. & isometry \\
\hline
\end{tabular}

Table 1. TextRank ordered lists.

The results show that asymmetric measures combined with directed graphs and graph-based ranking algorithms such as the TextRank are likely to give a positive answer to our hypothesis about the degree of generality of terms. Moreover, we propose an unsupervised methodology for acquiring general-specific noun relationships. However, it is clear that deep evaluation is needed.

\section{Experiments and Results}

Evaluation is classically a difficult task in Natural Language Processing. In fact, as human evaluation is time-consuming and generally subjective even when strict guidelines are provided, measures to automatically evaluate experiments must be proposed. In this section, we propose three evaluation measures and discuss the respective results.

\subsection{Constraints}

WordNet can be defined as applying a set of constraints to words. Indeed, if word $w$ is the hypernym of word $x$, we may represent this relation by the following constraint $y>x$, where $>$ is the order operator stating that $y$ is more general than $x$. As a consequence, for each set of three synsets (the hypernym synset, the seed synset and the hyponym synset), a list of constraints can be established i.e. all words of the hypernym synset must be more general than all the words of the seed synset and the hyponym synset, and all the words of the seed synset must be more general than all the words in the hyponym synset. So, if we take the synsets presented in Table 1, we can define the following set of constraints: \{rate $>$ growth rate, rate > rate of growth, growth rate > isometry, rate of growth > isometry\}.

In order to evaluate our list of words ranked by the level of generality against the WordNet categorization, we just need to measure the proportion of constraints which are respected as shown in Equation (10). We call, correctness this measure.

$$
\text { correctness }=\frac{\# \text { of common constraint }}{\# \text { of constraint }}
$$

For example, in Table 1, all the constraints are respected for both weighted and unweighted graphs, giving $100 \%$ correctness for the ordered lists compared to WordNet categorization.

\subsection{Clustering}

Another way to evaluate the quality of the ordering of words is to apply hard clustering to the words weighted by their level of generality. By evidencing the quality of the mapping between three hard clusters generated automatically and the hypernym synset, the seed synset and the hyponym synset, we are able to measure the quality of our ranking. As a consequence, we propose to (1) perform 3-means clustering over the list of ranked words, (2) classify the clusters by level of generality and (3) measure the precision, recall and f-measure of each cluster sorted by level of generality with the hypernym synset, the seed synset and the hyponym synset.

For the first task, we use the implementation of the k-means algorithm of the NLTK toolkit ${ }^{5}$. In particular, we bootstrap the k-means by choosing the initial means as follows. For the first mean, we choose the weight (the score) of the first word in the TextRank generated list of words. For the second mean, we take the weight of the middle word in the list and for the third mean, the weight of the last word in the list.

For the second task the level of generality of each cluster is evaluated by the average level of

\footnotetext{
${ }^{5}$ http://nltk.sourceforge.net/
} 
generality of words inside the cluster (or said with other words by its mean).

For the third task, the most general cluster and the hypernym synset are compared in terms ofprecision, recall and f-measure as shown in Equation (11), (12) and (13) 6 . The same process is applied to the second most general cluster and the seed synset, and the third cluster and the hyponym synset.

$$
\begin{gathered}
\text { precision }=\frac{\text { Cluster } \cap \text { Synset }}{\mid \text { Cluster } \mid} \\
\text { recall }=\frac{\text { Cluster } \cap \text { Synset }}{\mid \text { Synset } \mid} \\
f-\text { measure }=\frac{2 \times \text { recall } \times \text { precision }}{\text { precision }+ \text { recall }}
\end{gathered}
$$

\subsection{Rank Coefficient Test}

The evaluation can be seen as a rank test between two ordered lists. Indeed, one way to evaluate the results is to compare the list of generalspecific relationships encountered by the TextRank algorithm and the original list given by WordNet. However, we face one problem. WordNet does not give an order of generality inside synsets. In order to avoid this problem, we can order words in each synset by their estimated frequency given by WordNet $^{7}$ as well as their frequency calculated by web search hits. An example of both ordered lists is given in Table 2 for the synset \#6655336 and its immediate hypernyms and hyponyms.

\begin{tabular}{|c|c|c|c|}
\hline \multicolumn{2}{|c|}{ WordNet Estimated Frequency } & \multicolumn{2}{|c|}{ Web Estimated Frequency } \\
\hline Category & Word & Category & Word \\
\hline Hypernym & statement & Hypernym & statement \\
\hline Synset & answer & Synset & reply \\
\hline Synset & reply & Synset & response \\
\hline Synset & response & Synset & answer \\
\hline Hyponym & rescript & Hyponym & feedback \\
\hline Hyponym & feedback & Hyponym & rescript \\
\hline
\end{tabular}

Table 2. Estimated Frequency ordered lists for synset \#6655336.

For that purpose, we propose to use the Spearman's rank correlation coefficient (Rho). The Spearman's Rho is a statistical coefficient that shows how much two random variables are cor-

\footnotetext{
${ }^{6}$ Where Cluster $\cap$ Synset means the number of words common to both Synset and Cluster, and |Synset| and |Cluster| respectively measure the number of words in the Synset and the Cluster.

${ }^{7}$ We use WordNet 2.1.
}

related. It is defined in Equation (14) where $d$ is the distance between every pair of words in the list ordered with TextRank and the reference list which is ordered according to WordNet or the Web and $n$ is the number of pairs of ranked words.

$$
\rho=1-\frac{6 \times \sum d_{i}^{2}}{n\left(n^{2}-1\right)}
$$

In particular, the Spearman's rank correlation coefficient is a number between -1 (no correlation at all) and 1 (very strong correlation).

\subsection{Experiments}

In order to evaluate our methodology, we randomly $^{8}$ extracted 800 seed synsets for which we retrieved their hypernym and hyponym synsets. For each seed synset, we then built the associated directed weighted and unweighted graphs based on the asymmetric association measures referred to in section $2^{9}$ and ran the TextRank algorithm to produce a general-specific ordered lists of terms.

\subsubsection{Results by Constraints}

In Table 3, we present the results of the correctness for all seven asymmetric measures, both for the unweighted and weighted graphs.

\begin{tabular}{|c|c|c|}
\hline Equation & Type of Graph & Correctness \\
\hline \multirow{2}{*}{ Braun-Blanquet } & Unweighted & $65.68 \%$ \\
\cline { 2 - 3 } & Weighted & $65.52 \%$ \\
\hline \multirow{2}{*}{ J measure } & Unweighted & $60.00 \%$ \\
\cline { 2 - 3 } & Weighted & $60.34 \%$ \\
\hline \multirow{2}{*}{ Confidence } & Unweighted & $\mathbf{6 5 . 6 9 \%}$ \\
\cline { 2 - 3 } & Weighted & $65.40 \%$ \\
\hline \multirow{2}{*}{ Laplace } & Unweighted & $\mathbf{6 5 . 6 9 \%}$ \\
\cline { 2 - 3 } & Weighted & $\mathbf{6 5 . 6 9 \%}$ \\
\hline \multirow{2}{*}{ Conviction } & Unweighted & $61.81 \%$ \\
\cline { 2 - 3 } Certainty Factor & Weighted & $63.39 \%$ \\
\cline { 2 - 3 } & Unweighted & $65.59 \%$ \\
\hline \multirow{2}{*}{ Added Value } & Weighted & $63.76 \%$ \\
\cline { 2 - 3 } & Unweighted & $65.61 \%$ \\
\hline Baseline ${ }^{10}$ & Weighted & $64.90 \%$ \\
\hline \multirow{2}{*}{ Table 3. Resulted } & None & $55.68 \%$ \\
\hline
\end{tabular}

Table 3. Results for the Evaluation by Constraints.

The best results are obtained by the Confidence and the Laplace measures reaching $65.69 \%$ cor-

\footnotetext{
${ }^{8}$ We guarantee $98 \%$ significance level for an error of 0.05 following the normal distribution.

${ }^{9}$ The probability functions are estimated by the Maximum Likelihood Estimation (MLE).

${ }^{10}$ The baseline is the list of words ordered by web hits frequency (without TextRank).
} 
rectness. However, the Braun-Blanquet, the Certainty Factor and the Added Value give results near the best ones. Only the $\mathrm{J}$ measure and the Conviction metric seem to perform worst.

It is also important to note that the difference between unweighted and weighted graphs is marginal which clearly points at the fact that the topology of the graph is more important than its weighting. This is also confirmed by the fact that most of the asymmetric measures perform alike.

\subsubsection{Results by Clustering}

In Table 4, we present the results of precision, recall and f-measure for both weighted and unweighted graphs for all the seven asymmetric measures. The best precision is obtained for the weighted graph with the Confidence measure evidencing $47.62 \%$ and the best recall is also obtained by the Confidence measure also for the weighted graph reaching $47.68 \%$. Once again, the $\mathrm{J}$ measure and the Conviction metric perform worst showing worst f-measures. Contrarily, the Confidence measure shows the best performance in terms of f-measure for the weighted graph, i.e. $47.65 \%$ while the best result for the unweighted graphs is obtained by the Certainty factor with $46.50 \%$.

These results also show that the weighting of the graph plays an important issue in our methodology. Indeed, most metrics perform better with weighted graphs in terms of f-measure.

\begin{tabular}{|c|c|c|c|c|}
\hline Equation & Graph & Precision & Recall & F-measure \\
\hline \multirow{2}{*}{$\begin{array}{c}\text { Braun- } \\
\text { Blanquet }\end{array}$} & Unweighted & 46.61 & 46.06 & 46.33 \\
\cline { 2 - 5 } & Weighted & 47.60 & 47.67 & 47.64 \\
\hline \multirow{2}{*}{ J measure } & Unweighted & 40.92 & 40.86 & 40.89 \\
\cline { 2 - 5 } & Weighted & 42.61 & 43.71 & 43.15 \\
\hline \multirow{2}{*}{ Confidence } & Unweighted & 46.54 & 46.02 & 46.28 \\
\cline { 2 - 5 } & Weighted & $\mathbf{4 7 . 6 2}$ & $\mathbf{4 7 . 6 8}$ & $\mathbf{4 7 . 6 5}$ \\
\hline \multirow{2}{*}{ Laplace } & Unweighted & $\mathbf{4 6 . 6 7}$ & 46.11 & 46.39 \\
\cline { 2 - 5 } & Weighted & 46.67 & 46.11 & 46.39 \\
\hline \multirow{2}{*}{ Conviction } & Unweighted & 42.13 & 41.67 & 41.90 \\
\cline { 2 - 5 } & Weighted & 43.62 & 43.99 & 43.80 \\
\hline \multirow{2}{*}{$\begin{array}{c}\text { Certainty } \\
\text { Factor }\end{array}$} & Unweighted & 46.49 & $\mathbf{4 6 . 5 2}$ & $\mathbf{4 6 . 5 0}$ \\
\cline { 2 - 5 } & Weighted & 44.84 & 45.85 & 45.34 \\
\hline \multirow{2}{*}{$\begin{array}{c}\text { Added } \\
\text { Value }\end{array}$} & Unweighted & 46.61 & 46.59 & 46.60 \\
\cline { 2 - 5 } & Weighted & 47.13 & 47.27 & 47.19 \\
\hline
\end{tabular}

Table 4. Results for the Evaluation by Clustering.

In Table 5, 6 and 7, we present the same results as in Table 4 but at different levels of analysis i.e. precision, recall and f-measure at hypernym, seed and hyponym levels. Indeed, it is important to understand how the methodology performs at different levels of generality as we verified that our approach performs better at higher levels of generality.

\begin{tabular}{|c|c|c|c|c|}
\hline Equation & Graph & Precision & Recall & F-measure \\
\hline \multirow{2}{*}{$\begin{array}{c}\text { Braun- } \\
\text { Blanquet }\end{array}$} & Unweighted & 59.38 & 37.38 & 45.88 \\
\cline { 2 - 5 } & Weighted & 58.75 & 39.35 & 47.14 \\
\hline \multirow{3}{*}{ J measure } & Unweighted & 46.49 & 37.00 & 41.20 \\
\cline { 2 - 5 } & Weighted & 47.19 & 41.90 & 44.38 \\
\hline \multirow{3}{*}{ Confidence } & Unweighted & 59.20 & 37.30 & 45.77 \\
\cline { 2 - 5 } & Weighted & 58.71 & 39.22 & 47.03 \\
\hline \multirow{3}{*}{ Laplace } & Unweighted & $\mathbf{5 9 . 5 0}$ & 37.78 & $\mathbf{4 5 . 9 6}$ \\
\cline { 2 - 5 } & Weighted & $\mathbf{5 9 . 5 0}$ & 37.78 & 45.96 \\
\hline \multirow{3}{*}{$\begin{array}{c}\text { Conviction } \\
\text { Certainty } \\
\text { Factor }\end{array}$} & Unweighted & 50.07 & 35.88 & 41.80 \\
\cline { 2 - 5 } & Weighted & 52.72 & 40.74 & 45.96 \\
\cline { 2 - 5 } & Weighted & 51.64 & $\mathbf{4 2 . 9 3}$ & 46.88 \\
\hline \multirow{2}{*}{$\begin{array}{c}\text { Added } \\
\text { Value }\end{array}$} & Unweighted & 56.26 & 37.90 & 45.29 \\
\cline { 2 - 5 } & Weighted & 58.21 & 40.09 & $\mathbf{4 7 . 4 8}$ \\
\hline
\end{tabular}

Table 5. Results at the hypernym level.

\begin{tabular}{|c|c|c|c|c|}
\hline Equation & Graph & Precision & Recall & F-measure \\
\hline \multirow{2}{*}{$\begin{array}{c}\text { Braun- } \\
\text { Blanquet }\end{array}$} & Unweighted & 43.05 & 37.86 & 40.29 \\
\cline { 2 - 5 } & Weighted & $\mathbf{4 6 . 3 8}$ & 33.14 & 38.66 \\
\hline \multirow{2}{*}{ J measure } & Unweighted & 40.82 & $\mathbf{4 3 . 7 2}$ & 42.22 \\
\cline { 2 - 5 } & Weighted & 43.98 & 33.89 & 38.28 \\
\hline \multirow{2}{*}{ Confidence } & Unweighted & 43.03 & 37.67 & 40.17 \\
\cline { 2 - 5 } & Weighted & 46.36 & 33.02 & 38.57 \\
\hline \multirow{2}{*}{ Laplace } & Unweighted & 43.10 & 37.78 & 40.27 \\
\cline { 2 - 5 } & Weighted & 43.10 & 37.78 & 40.27 \\
\hline \multirow{2}{*}{ Conviction } & Unweighted & 40.36 & 38.02 & 39.16 \\
\cline { 2 - 5 } & Weighted & 42.60 & 26.39 & 32.59 \\
\hline \multirow{2}{*}{$\begin{array}{c}\text { Certainty } \\
\text { Factor }\end{array}$} & Unweighted & $\mathbf{4 4 . 2 8}$ & 40.87 & $\mathbf{4 2 . 5 1}$ \\
\cline { 2 - 5 } & Weighted & 44.14 & $\mathbf{4 0 . 7 0}$ & $\mathbf{4 2 . 3 5}$ \\
\hline \multirow{2}{*}{$\begin{array}{c}\text { Added } \\
\text { Value }\end{array}$} & Unweighted & 44.21 & 40.74 & 42.40 \\
\cline { 2 - 5 } & Weighted & 45.78 & 32.90 & 38.29 \\
\hline
\end{tabular}

Table 6. Results at the seed level.

\begin{tabular}{|c|c|c|c|c|}
\hline Equation & Graph & Precision & Recall & F-measure \\
\hline \multirow{2}{*}{$\begin{array}{c}\text { Braun- } \\
\text { Blanquet }\end{array}$} & Unweighted & 37.39 & 62.96 & 46.92 \\
\cline { 2 - 5 } & Weighted & 37.68 & 70.50 & 49.12 \\
\hline \multirow{2}{*}{ J measure } & Unweighted & 35.43 & 41.87 & 38.38 \\
\cline { 2 - 5 } & Weighted & 36.69 & 55.33 & 44.12 \\
\hline \multirow{2}{*}{ Confidence } & Unweighted & 37.38 & 63.09 & 46.95 \\
\cline { 2 - 5 } & Weighted & 37.79 & $\mathbf{7 0 . 8 0}$ & $\mathbf{4 9 . 2 7}$ \\
\hline \multirow{2}{*}{ Laplace } & Unweighted & 37.40 & $\mathbf{6 3 . 1 1}$ & 46.97 \\
\cline { 2 - 5 } & Weighted & 37.40 & 63.11 & 46.97 \\
\hline \multirow{2}{*}{ Conviction } & Unweighted & 35.97 & 50.94 & 42.16 \\
\cline { 2 - 5 } & Weighted & 35.54 & 64.85 & 45.92 \\
\hline \multirow{2}{*}{$\begin{array}{c}\text { Certainty } \\
\text { Factor }\end{array}$} & Unweighted & 39.28 & 60.40 & 47.60 \\
\cline { 2 - 5 } & Weighted & $\mathbf{3 8 . 7 4}$ & 53.92 & 45.09 \\
\hline \multirow{2}{*}{$\begin{array}{c}\text { Added } \\
\text { Value }\end{array}$} & Unweighted & $\mathbf{3 9 . 3 6}$ & 61.15 & $\mathbf{4 7 . 8 9}$ \\
\cline { 2 - 5 } & Weighted & 37.39 & 68.81 & 48.45 \\
\hline
\end{tabular}

Table 7. Results at the hyponym level.

Indeed, the precision scores go down from $59.50 \%$ at the hypernym level to $39.36 \%$ at the hyponym level with $46.38 \%$ at the seed level. The same phenomenon is inversely true for the recall with $42.93 \%$ at the hypernym level, 
$43.72 \%$ at the seed level and $70.80 \%$ at the hyponym level.

This situation can easily be understood as most of the clusters created by the k-means present the same characteristics i.e. the upper level cluster usually has fewer words than the middle level cluster which in turn has fewer words than the last level cluster. As a consequence, the recall is artificially high for the hyponym level. But on the opposite, the precision is high for higher levels of generality which is promising for the automatic construction of hierarchical thesauri. Indeed, our approach can be computed recursively so that each level of analysis is evaluated as if it was at the hypernym level, thus taking advantage of the good performance of our approach at upper levels of generality ${ }^{11}$.

\subsubsection{Results by Rank Test}

For each produced list, we calculated the Spearman's Rho both with WordNet and Web Estimated Lists for weighted and unweighted graphs. Table 8 presents the average results for the 800 randomly selected synsets.

\begin{tabular}{|c|c|c|c|}
\hline Equation & $\begin{array}{l}\text { Type of } \\
\text { Graph }\end{array}$ & $\begin{array}{c}\text { Rho with } \\
\text { WNet Est. } \\
\text { list }\end{array}$ & $\begin{array}{c}\text { Rho with } \\
\text { Web Est. } \\
\text { list }\end{array}$ \\
\hline \multirow{2}{*}{$\begin{array}{c}\text { Braun- } \\
\text { Blanquet }\end{array}$} & Unweighted & 0.38 & 0.30 \\
\hline & Weighted & 0.39 & 0.39 \\
\hline \multirow{2}{*}{$\mathrm{J}$ measure } & Unweighted & 0.23 & 0.19 \\
\hline & Weighted & 0.27 & 0.27 \\
\hline \multirow{2}{*}{ Confidence } & Unweighted & 0.38 & 0.30 \\
\hline & Weighted & 0.39 & 0.39 \\
\hline \multirow{2}{*}{ Laplace } & Unweighted & 0.38 & 0.30 \\
\hline & Weighted & 0.38 & 0.38 \\
\hline \multirow{2}{*}{ Conviction } & Unweighted & 0.30 & 0.22 \\
\hline & Weighted & 0.33 & 0.33 \\
\hline \multirow{2}{*}{$\begin{array}{c}\text { Certainty } \\
\text { Factor }\end{array}$} & Unweighted & 0.38 & 0.29 \\
\hline & Weighted & 0.35 & 0.35 \\
\hline \multirow{2}{*}{ Added Value } & Unweighted & 0.37 & 0.29 \\
\hline & Weighted & 0.38 & 0.38 \\
\hline Baseline $^{12}$ & None & 0.14 & 0.14 \\
\hline
\end{tabular}

Table 8. Results for the Spearman's rank correlation coefficient.

Similarly to what we evidenced in section 4.4.1., the $\mathrm{J}$ measure and the Conviction metric are the measures which less seem to map the correct order by evidencing low correlation scores. On the other hand, the Confidence metric still gives the best results equally with the Laplace and BraunBlanquet metrics.

\footnotetext{
${ }^{11}$ This will be studied as future work.

${ }^{12}$ The baseline is the list of words ordered by web hits frequency.
}

It is interesting to note that in the case of the web estimated list, the weighted graphs evidence much better results than the unweighted ones, although they do not show improved results compared to the WordNet list. On the one hand, these results show that our methodology is capable to map to WordNet lists as easily as to Web lists even that it is based on web frequency counts. On the other hand, the fact that weighted graphs perform best, shows that the topology of the graph lacks in accuracy and needs the application of weights to counterpoint this lack.

\subsection{Discussion}

An important remark needs to be made at this point of our explanation. There is a large ambiguity introduced in the methodology by just looking at web counts. Indeed, when counting the occurrences of a word like answer, we count all its occurrences for all its meanings and forms. For example, based on WordNet, the word answer can be a verb with ten meanings and a noun with five meanings. Moreover, words are more frequent than others although they are not so general, unconfirming our original hypothesis. Looking at Table 2, feedback is a clear example of this statement. As we are not dealing with a single domain within which one can expect to see the "one sense per discourse" paradigm, it is clear that the Rho coefficient would not be as good as expected as it is clearly biased by "incorrect" counts. One direct implication of this comment is the use of web estimated lists to evaluate the methodology.

Also, there has been a great discussion over the last few months in the corpora list ${ }^{13}$ whether one should use web counts instead of corpus counts to estimate word frequencies. In our study, we clearly see that web counts show evident problems, like the ones mentioned by (Kilgarriff, 2007). However, they cannot be discarded so easily. In particular, we aim at looking at web counts in web directories that would act as specific domains and would reduce the space for ambiguity. Of course, experiments with wellknown corpora will also have to be made to understand better this phenomenon.

\section{Conclusions and Future Work}

In this paper, we proposed a new methodology based on directed weighted/unweighted graphs and the TextRank algorithm to automatically in-

\footnotetext{
${ }^{13}$ Finalized by (Kilgarriff, 2007).
} 
duce general-specific noun relationships from web corpora frequency counts. To our knowledge, such an unsupervised experiment has never been attempted so far. In order to evaluate our results, we proposed three different evaluation metrics. The results obtained by using seven asymmetric association measures based on web frequency counts showed promising results reaching levels of (1) constraint coherence of $65.69 \%$, (2) clustering mapping of $59.50 \%$ in terms of precision for the hypernym level and $42.72 \%$ on average in terms of f-measure and (3) ranking similarity of 0.39 for the Spearman's rank correlation coefficient.

As future work, we intend to take advantage of the good performance of our approach at the hypernym level to propose a recursive process to improve precision results over all levels of generality.

Finally, it is important to notice that the evaluation by clustering evidences more than a simple evaluation of the word order, but shows how this approach is capable to automatically map clusters to WordNet classification.

\section{References}

Bollegala, D., Matsuo, Y. and Ishizuka, M. 2007. Measuring Semantic Similarity between Words Using WebSearch Engines. In Proceedings of International World Wide Web Conference (WWW 2007).

Caraballo, S.A. 1999. Automatic Construction of a Hypernym-labeled Noun Hierarchy from Text. In Proceedings of the Conference of the Association for Computational Linguistics (ACL 1999).

Dias, G., Santos, C., and Cleuziou, G. 2006. Automatic Knowledge Representation using a Graph-based Algorithm for Language-Independent Lexical Chaining. In Proceedings of the Workshop on Information Extraction Beyond the Document associated to the Joint Conference of the International Committee of Computational Linguistics and the Association for Computational Linguistics (COLING/ACL), pages. 36-47.

Grefenstette, G. 1994. Explorations in Automatic Thesaurus Discovery. Kluwer Academic Publishers, USA.

Hearst, M.H. 1992. Automatic Acquisition of Hyponyms from Large Text Corpora. In Proceedings of the Fourteenth International Conference on Com- putational Linguistics (COLING 1992), pages 539545.

Kilgarriff, A. 2007. Googleology is Bad Science. Computational Linguistics 33 (1), pages: 147-151.

Michelbacher, L., Evert, S. and Schütze, H. 2007. Asymmetric Association Measures. In Proceedings of the Recent Advances in Natural Language Processing (RANLP 2007).

Mihalcea, R. and Tarau, P. 2004. TextRank: Bringing Order into Texts. In Proceedings of the Conference on Empirical Methods in Natural Language Processing (EMNLP 2004), pages 404-411.

Pecina, P. and Schlesinger, P. 2006. Combining Association Measures for Collocation Extraction. In Proceedings of the International Committee of Computational Linguistics and the Association for Computational Linguistics (COLING/ACL 2006).

Riloff, E. 1993. Automatically Constructing a Dictionary for Information Extraction Tasks. In Proceedings of the Eleventh National Conference on Artificial Intelligence (AAAI 1993), pages 811-816.

Sang, E.J.K. and Hofmann, K. 2007. Automatic Extraction of Dutch Hypernym-Hyponym Pairs. In Proceedings of Computational Linguistics in the Netherlands Conference (CLIN 2007).

Snow, R., Jurafsky, D. and Ng, A. Y. 2005. Learning Syntactic Patterns for Automatic Hypernym Discovery. In Proceedings of the International Committee of Computational Linguistics and the Association for Computational Linguistics (COLING/ACL 2006).

Snow, R., Jurafsky, D. and Ng, A. Y. 2005. Semantic Taxonomy Induction from Heterogenous Evidence. In Proceedings of the Neural Information Processing Systems Conference (NIPS 2005).

Stevenson, M., and Greenwood, M. 2006. Comparing Information Extraction Pattern Models. In Proceedings of the Workshop on Information Extraction Beyond the Document associated to the Joint Conference of the International Committee of Computational Linguistics and the Association for Computational Linguistics (COLING/ACL 2006), pages. 29-35.

Tan, P.-N., Kumar, V. and Srivastava, J. 2004. Selecting the Right Objective Measure for Association Analysis. Information Systems, 29(4). pages 293313. 